\title{
Instrumentos de medida de qualidade de vida aplicados em indivíduos
}

\section{transplantados renais}

\author{
Quality of life measurement instruments applied to kidney transplanted individuals \\ Instrumentos de medición de la calidad de vida aplicados a personas trasplantadas de riñón
}

Recebido: 06/10/2021 | Revisado: 12/10/2021 | Aceito: 13/10/2021 | Publicado: 16/10/2021

\author{
Mariane Teixeira Dantas Farias \\ ORCID: https://orcid.org/0000-0003-4208-4911 \\ Universidade Federal da Bahia, Brasil \\ E-mail: marianedantas@bol.com.br \\ Edson Arakaki \\ ORCID: https://orcid.org/0000-0003-2184-2402 \\ Universidade Federal de São Paulo, Brasil \\ E-mail: edson.arakaki3020@gmail.com \\ Daniele Guedes Souza \\ ORCID: https://orcid.org/0000-0003-2184-2402 \\ Universidade Federal de São Paulo, Brasil \\ E-mail: danielle.guedes@uncisal.edu.br \\ Igor Ferreira Borba de Almeida \\ ORCID: https://orcid.org/0000-0002-8396-7385 \\ Universidade Federal da Bahia, Brasil \\ E-mail: borbadealmeidaigor@gmail.com \\ Bartira de Aguiar Roza \\ ORCID: https://orcid.org/0000-0002-6445-6846 \\ Universidade Federal de São Paulo, Brasil \\ E-mail: bartira.roza@unifesp.br \\ Elena Bohomol \\ ORCID: https://orcid.org/0000-0002-7196-0266 \\ Universidade Federal de São Paulo, Brasil \\ E-mail: ebohomol@unifesp.br \\ Regimar Carla Machado \\ ORCID: https://orcid.org/0000-0001-6126-7663 \\ Universidade Federal de São Paulo, Brasil \\ E-mail: regimar.carla@unifesp.br
}

\begin{abstract}
Resumo
O objetivo dessa pesquisa foi identificar na literatura os instrumentos aplicados para a mensuração da qualidade de vida (QV) em pacientes transplantados renais. Trata-se de uma revisão de literatura realizada nas bases de dados da Pubmed, Science direct e no portal da Biblioteca Virtual em Saúde (BVS) que identificou publicações que utilizaram Medidas de Resultados Relatados pelo Paciente (MRRP) no transplante renal. O corpus de análise dessa pesquisa foi obtido por 23 artigos, os quais apresentaram diversos instrumentos para avaliação da QV em transplantados renais. Foi possível constatar uma baixa produção bibliográfica desta temática, com uma média de 1,5 publicações ao ano, considerando o intervalo da amostra (1996-2021). O SF-36 foi o questionário predominante entre os estudos analisados, sendo os demais de abordagem genérica com características que divergiram entre os objetivos do avaliador e da pesquisa. Por fim, este estudo apontou para a necessidade de novas pesquisas que avaliem a aplicação de instrumentos mais específicos para esta população, para que assim sejam captadas as reais necessidades do transplantado renal, contribuindo para os cuidados centrados no paciente.
\end{abstract}

Palavras-chave: Transplante de rim; Questionário; Qualidade de vida.

\begin{abstract}
The objective of this research was to identify in the literature the instruments applied to measure the quality of life (QOL) in kidney transplant patients. This is a literature review carried out in the Pubmed, Science direct databases and in the Virtual Health Library (VHL) portal that identified publications that used Patient-Reported Outcome Measures (MRRP) in kidney transplantation. The corpus of analysis of this research was obtained by 23 articles, which presented different instruments for assessing QoL in kidney transplant recipients. It was possible to observe a low bibliographic production on this theme, with an average of 1.5 publications per year, considering the sample interval (1996-2021). The SF-36 was the predominant questionnaire among the analyzed studies, with the others having a generic approach with characteristics that diverged between the evaluator and the research objectives. Finally, this study pointed to the need for further research to assess the application of more specific instruments for
\end{abstract}


this population so that the real needs of kidney transplant recipients are captured, contributing to patient-centered care.

Keywords: Kidney transplatation; Quiz; Quality of life.

\section{Resumen}

El objetivo de esta investigación fue identificar en la literatura los instrumentos aplicados para medir la calidad de vida $(\mathrm{CV})$ en pacientes con trasplante renal. Se trata de una revisión de la literatura realizada en Pubmed, bases de datos Science direct y en el portal Virtual Health Library (VHL), que identificó publicaciones que utilizaron las medidas de resultado informadas por el paciente (MRRP) en el trasplante renal. El corpus de análisis de esta investigación se obtuvo mediante 23 artículos, que presentaron diferentes instrumentos para evaluar la CV en receptores de trasplante renal. Se pudo observar una baja producción bibliográfica sobre este tema, con un promedio de 1,5 publicaciones por año, considerando el intervalo muestral (1996-2021). El SF-36 fue el cuestionario predominante entre los estudios analizados, siendo los demás de abordaje genérico con características divergentes entre los objetivos del evaluador y los de la investigación. Finalmente, este estudio señaló la necesidad de profundizar en la investigación para evaluar la aplicación de instrumentos más específicos para esta población, de manera que se capten las necesidades reales de los receptores de trasplante renal, contribuyendo a la atención centrada en el paciente.

Palabras clave: Trasplante de riñón; Examen; Calidad de vida.

\section{Introdução}

O transplante renal é um procedimento de alta complexidade, que envolve a atuação de equipe multidisciplinar capacitada e uma infraestrutura hospitalar complexa, tornando-se uma alternativa de tratamento para a doença renal crônica (DRC) em fase avançada (Sharp, 1995; Wolfe et al., 1999). A terapêutica apresenta como principais objetivos, proporcionar a qualidade de vida e aumentar a sobrevida dos pacientes (Adeeb et al., 2021; Kamran \& Fife-Schaw, 2012; Muehrer \& Becker, 2005).

O Brasil possui o segundo maior programa de transplantes renais no mundo em números absolutos, atrás apenas dos Estados Unidos da América. Em 2019 foram realizados 6.417 transplantes renais, porém em 2020, devido à pandemia de COVID-19, houve uma queda de 24,5\% no número de transplantes renais no cenário brasileiro ("XXVI No 4 - Anual”, [s.d.]).

Para a Organização Mundial da Saúde (OMS), o conceito de qualidade de vida é a percepção do indivíduo, de sua inserção na vida, no contexto da cultura e sistemas de valores nos quais ele vive e em relação aos seus objetivos, expectativas, padrões e preocupações (“The World Health Organization Quality of Life Assessment (WHOQOL)”, 1995).

A qualidade de vida para indivíduos submetidos ao transplante renal é determinada por diversos fatores, que vão além da recuperação clínica e física. Envolve parâmetros de bem-estar físico, psicológico, emocional e social, que devido à subjetividade, muitas vezes tornam-se imensuráveis por instrumentos, escalas e questionários utilizados em pesquisas (Ware et al., 2019). Contudo, o transplante como terapia, reforça as medidas referentes aos critérios clínicos e laboratoriais, como mais relevantes para o acompanhamento das pessoas transplantadas (Adeeb et al., 2021; Baker et al., 2017).

Os questionários mais utilizados para a avaliação da qualidade de vida em pacientes com doença renal crônica são o Kidney Disease and Quality of Life-Short Form (KDQOL-SF) e Chronic Kidney Disease Quality of Life (CKD-QOL) derivado do Health Survey Short Form 36 (SF-36) e suas abreviações como o Health Survey Short Form 12 (SF-12) (Ware et al., 2019). Tais instrumentos são baseados nas Medidas de Resultados Relatados pelo Paciente (MRRP), que constituem mais uma ferramenta de acompanhamento do paciente nos pós transplante, pois conseguem traduzir sua real condição, uma vez que as informações são coletadas diretamente destes, sem a interpretação de terceiros (Agochukwu et al., 2020).

Diante de transformações significativas nos últimos anos referentes à concepção dos conceitos de saúde e doença, vivenciados pelo paciente, emergiram inquietações que permeiam a aplicação de questionários e as características que os compõem, à medida que se tem problematizado a mensuração da qualidade de vida. Observa-se a necessidade de ampla discussão acerca de instrumentos que são aplicados para a avaliação da qualidade de vida, com base em métodos relevantes e que atendam às necessidades de pessoas pós transplantadas. 
Esta pesquisa se justifica, numa perspectiva científica, pelo limitado número de estudos que abordam a temática, e na lacuna existente referente às MRRP em transplantados renais (Verberne et al., 2019). Considerando que, além da sobrevida, a qualidade de vida é prioridade a ser alcançada por pacientes transplantados renais (Wu et al., 2010), justifica-se a necessidade de aprofundar o conhecimento acerca da temática que envolve os instrumentos e/ou questionários que mensuram este aspecto. O objetivo deste estudo foi identificar na literatura os instrumentos aplicados para a mensuração da qualidade de vida em pacientes transplantados renais.

\section{Materiais e Método}

Trata-se de uma revisão da literatura, delimitada em seu primeiro momento pela identificação da temática e, em seguida pela seleção da pergunta de pesquisa. Isto posto, procedeu-se às demais etapas, tais como definição dos critérios de inclusão e exclusão, seleção da amostra, análise e discussão dos achados até atingir a síntese do conhecimento (Soares et al., 2014).

Para a construção da pergunta de revisão foi utilizado o mnemônico "PICO", no qual a "População" foi o transplantado renal, "Interesse" referiu-se às medidas de resultados relatadas pelo paciente, o "Contexto" ao uso de questionários e/ou instrumentos e os "Outcomes" (resultados) a qualidade de vida. Para conduzir este estudo foi adotada a seguinte questão: “Quais são os instrumentos de medida de qualidade de vida utilizados em indivíduos transplantados renais?”.

Neste sentido, conduziu-se uma busca inicial, realizada entre os meses de julho e agosto de 2021, em periódicos indexados nas bases de dados da Pubmed, Sciencedirect e no portal da Biblioteca Virtual em Saúde (BVS). Primeiramente, foi realizada leitura na ordem de título, resumo, palavras-chave/descritores. Os estudos elencados que responderam à questão de pesquisa foram lidos na íntegra e as suas referências analisadas em busca de estudos adicionais. Os termos utilizados em cada base selecionada foram especificados e definidos após a primeira busca.

A estratégia de busca para identificação e seleção dos estudos foi estruturada com os descritores, por meio dos indicadores booleanos "and" e "or" da seguinte forma: (Transplante de Rim OR Kidney Transplantation OR Renal Transplantation) AND (Medidas de Resultados Relatados pelo Paciente OR Patient-Reported Outcome Measures) AND (Quiz OR Questionnaire) AND (Qualidade de Vida OR Quality of Life).

Sobre os critérios de inclusão, fontes de informação e pesquisa, os periódicos considerados se apresentaram nos idiomas português, inglês e espanhol, sob a forma de texto completo que resultaram de estudos que aplicaram ou validaram instrumentos de mensuração da qualidade de vida no pós-transplante renal, em maiores de 18 anos. Não houve a delimitação do tempo, considerando o escopo de uma revisão da literatura.

Foram excluídas as publicações que não estavam disponíveis para download, as identificadas em duplicata, desvinculadas à temática do estudo, estudos de revisão (bibliométricos, revisão sistemática e integrativa) e que foram realizados com animais ou crianças. Três revisores independentes realizaram a seleção dos artigos com base no título e nos resumos, após a busca, não existindo discordância entre estes.

O processo de busca e seleção dos estudos dessa revisão está apresentado na Figura 1, adaptado do Preferred Reporting Items for Systematic reviews and Meta-Analyses extension for Scoping Reviews (PRISMA-ScR) (Liberati et al., 2009). 
Figura 1 - Fluxograma do processo de busca e seleção dos estudos utilizada para revisão integrativa, adaptado do PRISMA. São Paulo-SP, 2021.

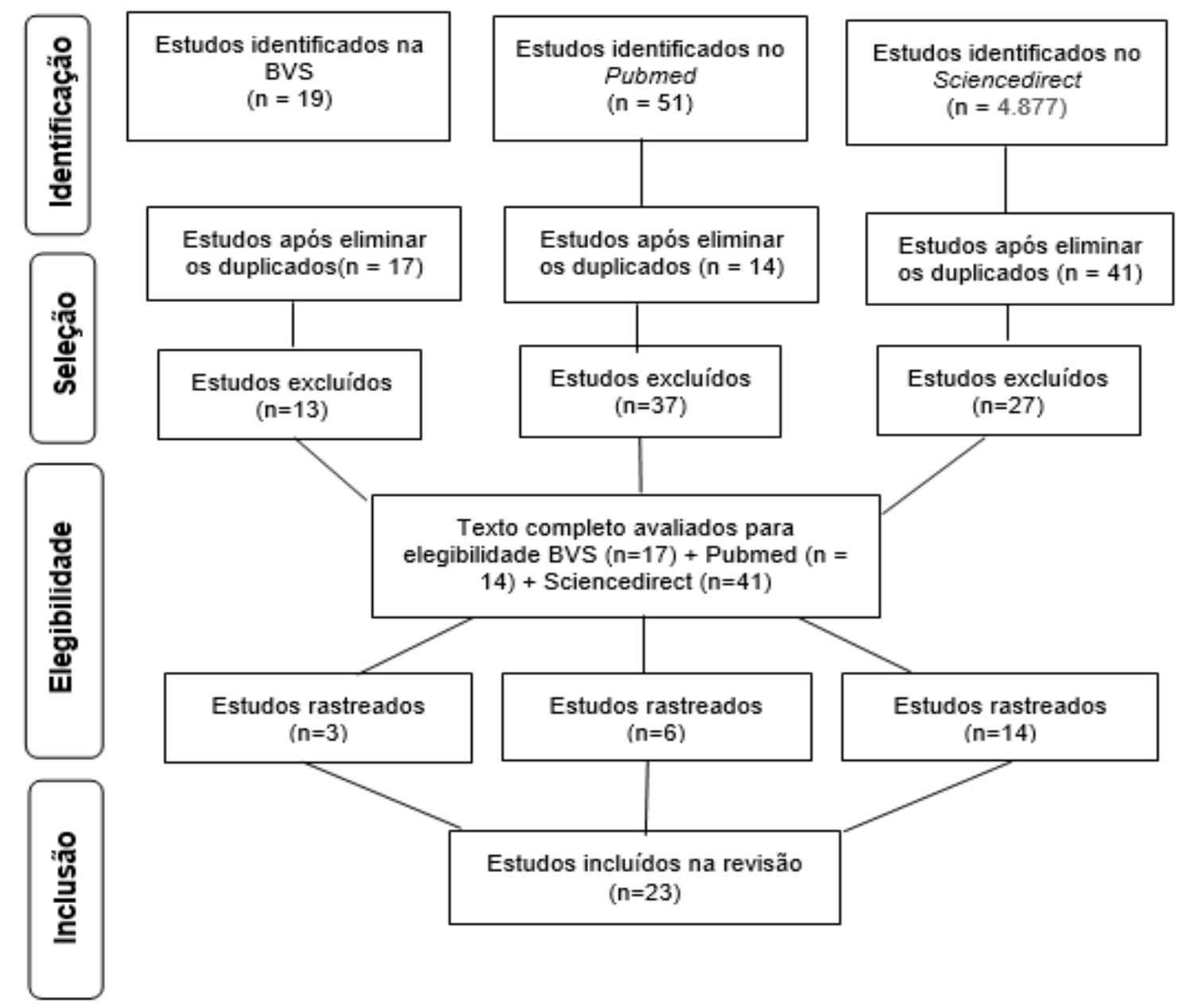

Fonte: Autores (2021).

É importante considerar que os dados dos estudos elegidos foram extraídos por meio de um formulário estruturado pelos pesquisadores, o qual contemplou: título do estudo, base indexada, ano de publicação, local do estudo (país), detalhamento metodológico, principais resultados, instrumento utilizado para avaliar a QV.

\section{Resultados}

A análise dos dados mostrou que a ScienceDirect é uma base de busca mais abrangente ter captado um maior quantitativo de estudos, quando comparada ao portal BVS e ao PubMed, utilizando-se a mesma estratégia de busca. Isto posto, inferiu-se que o portal BVS e a PubMed possuem mecanismos de filtragem mais eficientes para delimitação dos estudos (Quadro 1). 
Quadro 1 - Apresentação dos resultados da pesquisa. São Paulo, SP, Brasil, 2021.

\begin{tabular}{|c|c|c|c|c|c|c|}
\hline $\begin{array}{c}\text { Base de } \\
\text { Dados }\end{array}$ & Título do artigo & $\begin{array}{c}\text { Ano de } \\
\text { Publicação }\end{array}$ & $\begin{array}{l}\text { País onde } \\
\text { foi } \\
\text { realizado o } \\
\text { estudo }\end{array}$ & Objetivo do estudo & Principais resultados & $\begin{array}{c}\text { Tipo de } \\
\text { Questionário }\end{array}$ \\
\hline $\begin{array}{l}\text { ScienceDi } \\
\text { rect }\end{array}$ & $\begin{array}{l}\text { Um estudo da } \\
\text { qualidade de } \\
\text { vida e custo- } \\
\text { utilidade do } \\
\text { transplante renal } \\
\text { (Laupacis et al., } \\
\text { 1996) }\end{array}$ & 1996 & Canadá & $\begin{array}{l}\text { Avaliaro custo- } \\
\text { utilidade do transplante } \\
\text { renal em comparação } \\
\text { com a diálise. }\end{array}$ & $\begin{array}{l}\text { Melhora dos escores médios de QV em } \\
\text { quase todos os aspectos em seis meses } \\
\text { pós-tx comparado ao pré-tx, } \\
\text { permanecendo melhores ao longo dos } \\
\text { dois anos de acompanhamento. }\end{array}$ & $\begin{array}{l}\text { Chronic Kidney } \\
\text { Disease Quality of } \\
\text { Life (CKD-QOL) }\end{array}$ \\
\hline Pubmed & $\begin{array}{l}\text { Diferenças de } \\
\text { raça e gênero na } \\
\text { qualidade de } \\
\text { vida após o } \\
\text { transplante renal } \\
\text { (Johnson et al., } \\
\text { 1998) }\end{array}$ & 1998 & $\begin{array}{l}\text { Estados } \\
\text { Unidos }\end{array}$ & $\begin{array}{l}\text { Determinar se a raça ou } \\
\text { gênero afeta mudanças } \\
\text { na qualidade de vida } \\
\text { (QoL) relatada por } \\
\text { transplantados renais } \\
\text { não diabéticos }\end{array}$ & $\begin{array}{l}\text { Afro-americanos com índices menores } \\
\text { em relação aos americanos caucasianos } \\
\text { quanto às medidas afetivas e funcionais } \\
\text { da QV. As mulheres pontuaram menos } \\
\text { que os homens na maioria das medidas } \\
\text { de QV no período basal, porém } \\
\text { relataram melhora na capacidade } \\
\text { funcional. A percepção da auto-imagem } \\
\text { permaneceu baixa. }\end{array}$ & $\begin{array}{l}\text { Perfil do Impacto da } \\
\text { Doença } \\
\text { Índice de Ferrans } \\
\text { and Powers de QV } \\
\text { Escalas de Auto- } \\
\text { Imagem para } \\
\text { adultos. }\end{array}$ \\
\hline $\begin{array}{l}\text { ScienceDi } \\
\text { rect }\end{array}$ & $\begin{array}{l}\text { Qualidade de } \\
\text { vida, } \\
\text { enfrentamento e } \\
\text { preocupações em } \\
\text { pacientes } \\
\text { chineses (Kong } \\
\text { \& Molassiotis, } \\
\text { 1999) }\end{array}$ & 1999 & China & $\begin{array}{l}\text { Identificar os métodos } \\
\text { de enfrentamento } \\
\text { utilizados por pacientes } \\
\text { para lidar com o } \\
\text { estresse após o } \\
\text { transplante renal e } \\
\text { avaliar a percepção da } \\
\text { qualidade de vida dos } \\
\text { pacientes em dois } \\
\text { intervalos de tempo } \\
\text { diferentes após o } \\
\text { transplante. }\end{array}$ & $\begin{array}{l}\text { Não houve significânciaestatistica na } \\
\text { utilização do CCS em } \\
\text { medir o enfrentamento dos pacientes } \\
\text { dentro de um ano ou mais após o } \\
\text { transplante, sendo utilizado o locus } \\
\text { interno de enfrentamento do estresse. } \\
\text { Não foram encontradas } \\
\text { diferenças estatísticas em todas as } \\
\text { subescalas de QV entre os dois grupos } \\
\text { de } \\
\text { pacientes. A QV foi moderada nos } \\
\text { pacientes de tx renal em H. Kong }\end{array}$ & $\begin{array}{l}\text { A escala chinesa de } \\
\text { enfrentamento } \\
\text { (CCS) } \\
\text { Escala WHOQOL } \\
\text { (versão chinesa) }\end{array}$ \\
\hline $\begin{array}{l}\text { ScienceDi } \\
\text { rect }\end{array}$ & $\begin{array}{l}\text { Mudança na } \\
\text { qualidade de } \\
\text { vida após o } \\
\text { transplante renal } \\
\text { (Ponton et al., } \\
\text { 2001) }\end{array}$ & 2001 & Itália & $\begin{array}{l}\text { avaliar o estado físico e } \\
\text { psicológico dos pts, } \\
\text { comparar aspectos } \\
\text { objetivos e subjetivos } \\
\text { da QV, e as correlações } \\
\text { entre aspectos } \\
\text { somáticos, psicológicos } \\
\text { e sociais. }\end{array}$ & $\begin{array}{l}\text { Houve melhora nos indicadores de QV } \\
\text { pós-transplante a curto e longo prazo, } \\
\text { porém, entre ( } 7 \text { a } 36 \text { meses pós tx) } \\
\text { houve piora retornando aos índices pré } \\
\text { tx. Os resultados do LEIPAD, oferecem } \\
\text { uma ideia melhor da experiência } \\
\text { emocional do transplante influencia } \\
\text { fortemente o resultado em termos da } \\
\text { QV percebida. }\end{array}$ & TOTLEIPAD \\
\hline $\begin{array}{l}\text { ScienceDi } \\
\text { rect }\end{array}$ & $\begin{array}{l}\text { Impacto do } \\
\text { status } \\
\text { socioeconômico } \\
\text { na qualidade de } \\
\text { vida de Pacientes } \\
\text { ESRD (Sesso et } \\
\text { al., 2003) }\end{array}$ & 2003 & Brasil & $\begin{array}{l}118 \text { pacientes foram } \\
\text { avaliados } \\
\text { prospectivamente no } \\
\text { início do tratamento } \\
\text { dialítico e reavaliados } \\
\text { (n 90) após uma média } \\
\text { de } 7 \text { meses. }\end{array}$ & $\begin{array}{l}\text { Obteve pontuações médias reduzidas } \\
\text { para as dimensões do SF-36 em } \\
\text { pacientes de baixo SES comparados aos } \\
\text { de alto, com maiores diferenças } \\
\text { observadas durante o acompanhamento. } \\
\text { O SES afetou significativamente todas } \\
\text { as dimensões da QV, explicando 5,5\% a } \\
14,1 \% \text { de variação em escalas. }\end{array}$ & $\begin{array}{l}\text { Short Form } 36 \\
\text { Health Survey }\end{array}$ \\
\hline
\end{tabular}


Research, Society and Development, v. 10, n. 13, e337101321548, 2021

(CC BY 4.0) | ISSN 2525-3409 | DOI: http://dx.doi.org/10.33448/rsd-v10i13.21548

\begin{tabular}{|c|c|c|c|c|c|c|}
\hline $\begin{array}{l}\text { ScienceDi } \\
\text { rect }\end{array}$ & $\begin{array}{l}\text { Efeitos de } \\
\text { diabetes e órgãos } \\
\text { cadavéricos no } \\
\text { desempenho } \\
\text { funcional e } \\
\text { qualidade de } \\
\text { vida relacionada } \\
\text { à saúde após o } \\
\text { transplante } \\
\text { renal.(Kizilisik } \\
\text { et al., 2003) }\end{array}$ & 2003 & $\begin{array}{l}\text { Estados } \\
\text { Unidos }\end{array}$ & $\begin{array}{l}\text { Identificar fatores } \\
\text { específicos para o } \\
\text { transplante renal } \\
\text { associados ao } \\
\text { desempenho funcional } \\
\text { pós-transplante (FP) e à } \\
\text { qualidade de vida } \\
\text { relacionada à saúde } \\
\text { (QVRS). }\end{array}$ & $\begin{array}{l}\text { FP melhorou após o transplante renal } \\
\text { (em } 0,3,6 \text { e } 12 \text { meses, } \\
\text { respectivamente). Os transplantados de } \\
\text { doadores vivos mostraram melhoria } \\
\text { contínua em } 1 \text { ano pós-tx, enquanto os } \\
\text { de cadáveres estabilizaram no mês } 6 \\
\text { (contraste de interação simples, ano } 1 \\
\text { versus pré-tx). Pacientes dialíticos por } 6 \\
\text { meses ou mais no pre-tx demonstraram } \\
\text { escores de FP pós-tx mais baixos do SF- } \\
36 \text { em quando comparados aos TX } \\
\text { preventivamente. Por sua vez, o FP teve } \\
\text { um efeito direto positivo na QVRS. }\end{array}$ & $\begin{array}{l}\text { Short Form } 36 \\
\text { Health Survey }\end{array}$ \\
\hline BVS & $\begin{array}{l}\text { Um estudo } \\
\text { comparativo da } \\
\text { qualidade de } \\
\text { vida entre as } \\
\text { faixas etárias de } \\
\text { receptores de } \\
\text { transplante } \\
\text { renal.(Cetingok } \\
\text { et al., 2004) }\end{array}$ & 2004 & $\begin{array}{l}\text { Estados } \\
\text { Unidos }\end{array}$ & $\begin{array}{l}\text { Determinar se existem } \\
\text { diferenças na qualidade } \\
\text { de vida pré e pós- } \\
\text { transplante de } \\
\text { receptores de } \\
\text { transplante renal em } \\
\text { relação à idade. }\end{array}$ & $\begin{array}{l}\text { A QV pré e pós-tx de todas as faixas } \\
\text { etárias dos receptores de tx renal foram } \\
\text { semelhantes, exceto os receptores na } \\
\text { faixa etária de } 30-49 \text { anos que relataram } \\
\text { melhor } \mathrm{QV} \text { pós-transplante na medida } \\
\text { psicossocial do Perfil de Impacto da } \\
\text { Doença ( } \mathrm{F}=2,18, \mathrm{P}=0,02 \text { ). }\end{array}$ & $\begin{array}{l}\text { Índice de Qualidade } \\
\text { de Vida; } \\
\text { Escala de } \\
\text { Autoimagem para } \\
\text { Adultos; } \\
\text { Perfil de Impacto da } \\
\text { Doença }\end{array}$ \\
\hline $\begin{array}{l}\text { ScienceDi } \\
\text { rect }\end{array}$ & $\begin{array}{l}\text { A importância da } \\
\text { satisfação do } \\
\text { paciente e da } \\
\text { saúde } \\
\text { relacionada a } \\
\text { QV após o TX } \\
\text { renal (Yildirim, } \\
\text { 2006) }\end{array}$ & 2006 & Turquia & $\begin{array}{l}\text { Examinar e comparar a } \\
\text { qualidade de vida e a } \\
\text { satisfação em pacientes } \\
\text { transplantados renais } \\
\text { com doença renal em } \\
\text { estágio terminal. }\end{array}$ & $\begin{array}{l}\text { Os escores médios de satisfação do } \\
\text { paciente (PSQ III) e qualidade de vida } \\
\text { (15D) foram melhores entre os } \\
\text { pacientes de tx renal masculino do que } \\
\text { os pacientes em diálise ou peritoneal. A } \\
\text { QV de pacientes de tx casados e a } \\
\text { satisfação obtiveram pontuações médias } \\
\text { significativamente maiores do que os } \\
\text { solteiros ou viúvos. Os escores totais no } \\
\text { PSQ III mostraram uma correlação } \\
\text { positiva significativa com os escores } \\
\text { totais do 15D. }\end{array}$ & $\begin{array}{l}\text { 15D } \\
\text { PSQ III }\end{array}$ \\
\hline $\begin{array}{l}\text { ScienceDi } \\
\text { rect }\end{array}$ & $\begin{array}{l}\text { Qualidade de } \\
\text { vida específica, } \\
\text { em pacientes } \\
\text { turcos após o } \\
\text { transplante renal } \\
\text { bem-sucedido } \\
\text { (Franke et al., } \\
\text { 2006). }\end{array}$ & 2006 & Turquia & $\begin{array}{l}\text { Avaliar o instrumento } \\
\text { de QV ESRD-SCL-TM } \\
\text { específico da doença } \\
\text { em pacientes turcos. }\end{array}$ & $\begin{array}{l}\text { Os pacientes turcos tiveram } \\
\text { significativamente mais sofrimento } \\
\text { específico da doença do que os } \\
\text { pacientes alemães, em relação a quatro } \\
\text { das seis subescalas: Capacidade física } \\
\text { limitada, Capacidade cognitiva limitada, } \\
\text { Efeitos colaterais dos corticosteróides e } \\
\text { Angústia psicológica associada ao } \\
\text { transplante. Em relação ao escore } \\
\text { global, houve significativamente mais } \\
\text { sofrimento específico da doença. }\end{array}$ & $\begin{array}{l}\text { End-Stage Renal } \\
\text { Disease Symptom } \\
\text { Checklist } \\
\text { Transplantation } \\
\text { Module } \\
\text { (ESRDSCL-TM) }\end{array}$ \\
\hline $\begin{array}{l}\text { ScienceDi } \\
\text { rect }\end{array}$ & $\begin{array}{l}\text { Qualidade de } \\
\text { vida e fatores } \\
\text { psicossociais } \\
\text { renais } \\
\text { destinatários do } \\
\text { transplante } \\
\text { (Shah et al., } \\
\text { 2006) }\end{array}$ & 2006 & $\begin{array}{l}\text { Estados } \\
\text { Unidos }\end{array}$ & $\begin{array}{l}\text { Avaliar receptores de } \\
\text { transplante renal (RTR) } \\
\text { usando vários } \\
\text { questionários } \\
\text { psicossociais. Nós } \\
\text { hipotetizamos que a } \\
\text { percepção dos } \\
\text { pacientes sobre a } \\
\text { depressão, carga de } \\
\text { doença, apoio social e } \\
\text { satisfação com a vida } \\
\text { não se correlacionam } \\
\text { com sua percepção de } \\
\text { QV. }\end{array}$ & $\begin{array}{l}\text { Dos pacientes, } 64 \% \text { eram afro- } \\
\text { americanos e } 48 \% \text { eram mulheres; } 94 \% \\
\text { dos pacientes tiveram pontuação 5. O } \\
\text { QLS médio foi de 7,5 a 2,3. Percepção } \\
\text { de uma melhor QV correlacionada com } \\
\text { menos percepção dos efeitos da } \\
\text { depressão e da doença e com percepção } \\
\text { de maior suporte social e satisfação com } \\
\text { a vida. }\end{array}$ & $\begin{array}{l}\text { Escore da escala de } \\
\text { QV (QLS) }\end{array}$ \\
\hline
\end{tabular}


Research, Society and Development, v. 10, n. 13, e337101321548, 2021

(CC BY 4.0) | ISSN 2525-3409 | DOI: http://dx.doi.org/10.33448/rsd-v10i13.21548

\begin{tabular}{|c|c|c|c|c|c|c|}
\hline $\begin{array}{l}\text { ScienceDi } \\
\text { rect }\end{array}$ & $\begin{array}{l}\text { Validação do } \\
\text { KDQoL-Short } \\
\text { Form em } \\
\text { pacientes } \\
\text { transplante renal } \\
\text { (Barotfi et al., } \\
\text { 2006) }\end{array}$ & 2006 & Hungria & $\begin{array}{l}\text { Determinar as } \\
\text { propriedades } \\
\text { psicométricas básicas, } \\
\text { confiabilidade e } \\
\text { validade do } \\
\text { questionário Kidney } \\
\text { Disease Quality of } \\
\text { Life-Short (KDQOL- } \\
\text { SF) em pacientes } \\
\text { transplantados renais. }\end{array}$ & $\begin{array}{l}\text { A consistência interna de todos os } \\
\text { domínios do Medical Outcome Study } \\
\text { Short Form } 36 \text { (SF-36) mostrou-se } \\
\text { muito boa, e o valor alfa de Cronbach } \\
\text { estava acima de } 0,70 \text { para todas, exceto } \\
\text { para três subescalas específicas da } \\
\text { doença. Houve diferenças substanciais } \\
\text { no escore do KDQOL-SF observadas } \\
\text { entre grupos de pacientes Tx que } \\
\text { deveriam ser clinicamente diferentes, } \\
\text { apoiando a validade discriminante do } \\
\text { instrumento. }\end{array}$ & $\begin{array}{l}\text { Short Form } 36 \\
\text { Health Survey }\end{array}$ \\
\hline Pubmed & $\begin{array}{l}\text { O excesso de } \\
\text { peso do pré- } \\
\text { transplante e a } \\
\text { obesidade não } \\
\text { afetam a } \\
\text { qualidade física } \\
\text { da vida após o } \\
\text { transplante renal } \\
\text { (Zaydfudim et } \\
\text { al., 2010) }\end{array}$ & 2010 & $\begin{array}{l}\text { Estados } \\
\text { Unidos }\end{array}$ & $\begin{array}{l}\text { Examinar os efeitos do } \\
\text { excesso de peso pré-tx } \\
\text { e e obesidade na Saúde } \\
\text { Relacionada à } \\
\text { Qualidade de Vida } \\
\text { (HRQOL), pós-tx em } \\
\text { receptores de tx renal }\end{array}$ & $\begin{array}{l}\text { Os receptores de tx renal que não } \\
\text { exigiram diálise pré-transplante e } \\
\text { aqueles que foram manejados evitando } \\
\text { corticosteróides após o tx, foram os } \\
\text { mais propensos a alcançar pontuações } \\
\text { acima da média ou a mesma da } \\
\text { população geral. }\end{array}$ & $\begin{array}{l}\text { Short Form } 36 \\
\text { Health Survey }\end{array}$ \\
\hline Pubmed & $\begin{array}{l}\text { O efeito de uma } \\
\text { intervenção } \\
\text { educacional para } \\
\text { transplantados } \\
\text { renais: um } \\
\text { ensaio } \\
\text { controlado } \\
\text { randomizado } \\
\text { (Urstad et al., } \\
\text { 2012) }\end{array}$ & 2012 & Noruega & $\begin{array}{l}\text { Testar a eficácia de } \\
\text { uma intervenção } \\
\text { educacional sobre o } \\
\text { conhecimento, a } \\
\text { conformidade, a auto- } \\
\text { eficácia, a qualidade de } \\
\text { vida do Transplantado } \\
\text { renal. }\end{array}$ & $\begin{array}{l}\text { Encontrou-se níveis significativos mais } \\
\text { altos de conhecimento no grupo } \\
\text { experimental comparado ao grupo } \\
\text { controle, bem como sobre a autoeficácia } \\
\text { e no escore mental de qualidade de vida. }\end{array}$ & 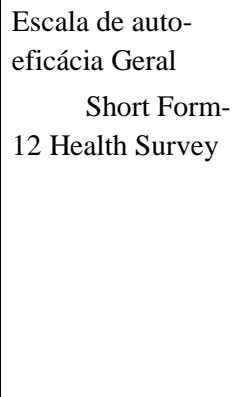 \\
\hline Pubmed & $\begin{array}{l}\text { Da diálise ao } \\
\text { transplante: um } \\
\text { estudo } \\
\text { longitudinal de } 5 \\
\text { anos sobre a } \\
\text { qualidade de } \\
\text { vida } \\
\text { autorreferida } \\
\text { (von der Lippe et } \\
\text { al., 2014) }\end{array}$ & 2014 & Noruega & $\begin{array}{l}\text { Para avaliar a Saúde } \\
\text { Relacionada à } \\
\text { Qualidade de Vida } \\
\text { (HRQOL) em pacientes } \\
\text { de diálise, que } \\
\text { seguiram para } \\
\text { Transplante renal } \\
\text { (RTX). Além disso, } \\
\text { comparar a HRQOL } \\
\text { em pacientes com RTX } \\
\text { e a população em geral. }\end{array}$ & $\begin{array}{l}\text { Melhorias significativas na Saúde } \\
\text { Relacionada à QV (HRQoL) foram } \\
\text { observadas após o Tx renal em vários } \\
\text { dos domínios da HRQOL específicos do } \\
\text { rim. Efeitos do ônus da doença renal } \\
\text { como, sintomas, sono, função sexual e } \\
\text { trabalho, melhoraram, enquanto a } \\
\text { função cognitiva, o apoio social e a } \\
\text { qualidade da interação social não. }\end{array}$ & $\begin{array}{l}\text { Kidney Disease and } \\
\text { Quality of Life- } \\
\text { Short Form } \\
\text { (KDQOL-SF) } \\
\text { Short Form-SF } 36 \\
\text { Health Survey) }\end{array}$ \\
\hline BVS & $\begin{array}{l}\text { Efeito benéfico } \\
\text { do belatacept na } \\
\text { QV relacionada } \\
\text { à saúde e efeitos } \\
\text { colaterais } \\
\text { percebidos: } \\
\text { resultados dos } \\
\text { estudos } \\
\text { BENEFIT e } \\
\text { BENEFIT- } \\
\text { EXT.(Dobbels et } \\
\text { al., 2014) }\end{array}$ & 2014 & Bélgica & $\begin{array}{l}\text { Investigar: (a) a } \\
\text { evolução da QVRS, } \\
\text { avaliada pelo SF-36 } \\
\text { nos primeiros } 3 \text { anos } \\
\text { (linha de base, 12, } 24 \text { e } \\
36 \text { meses) após o tx } \\
\text { renal; (b) associação } \\
\text { entre fç renal-estágio } \\
\text { de DRC, QVRS e } \\
\text { efeitos colaterais } \\
\text { relatados pelo paciente } \\
\text { (EOSTM e Angústia de } \\
\text { Sintomas-59R) e (c) } \\
\text { impacto do belatacepte } \\
\text { e da ciclosporina na } \\
\text { experiência de efeitos } \\
\text { colaterais e na QVRS. }\end{array}$ & $\begin{array}{l}\text { No estudo BENEFIT, todos os } \\
\text { indivíduos relataram melhorias } \\
\text { clinicamente significativas em } \\
\text { comparação com a linha de base e } \\
\text { retornaram aos escores da população } \\
\text { geral (SF 36), entre } 12 \text { a } 36 \text { meses após } \\
\text { o tx. Os pacientes tratados com } \\
\text { belatacept relataram melhores PCSs } \\
\text { absolutos em comparação com os } \\
\text { pacientes tratados com ciclosporina. A } \\
\text { piora da função renal foi associada a } \\
\text { uma redução significativa na QVRS. }\end{array}$ & $\begin{array}{l}\text { Short Form } 36 \\
\text { Health Survey; } \\
\quad \text { Escala de } \\
\text { Ocorrência de } \\
\text { Sintomas de } \\
\text { Transplante } \\
\text { Modificada; } \\
\text { Angústia de } \\
\text { Sintomas-59R. }\end{array}$ \\
\hline
\end{tabular}


Research, Society and Development, v. 10, n. 13, e337101321548, 2021

(CC BY 4.0) | ISSN 2525-3409 | DOI: http://dx.doi.org/10.33448/rsd-v10i13.21548

\begin{tabular}{|c|c|c|c|c|c|c|}
\hline $\begin{array}{l}\text { ScienceDi } \\
\text { rect }\end{array}$ & $\begin{array}{l}\text { Um estudo sobre } \\
\text { adesão, } \\
\text { qualidade de } \\
\text { vida e fatores } \\
\text { associados entre } \\
\text { os receptores de } \\
\text { transplantes } \\
\text { renais na China } \\
\text { (Zhao et al., } \\
\text { 2017) }\end{array}$ & 2017 & China & $\begin{array}{l}\text { Investigamos o status } \\
\text { de adesão ao } \\
\text { acompanhamento e QV } \\
\text { e fatores associados } \\
\text { entre receptores de } \\
\text { transplante renal na } \\
\text { China. }\end{array}$ & $\begin{array}{l}\text { A pontuação média para adesão ao } \\
\text { acompanhamento foi de } 54,71+-6,46 \text {. } \\
\text { O tempo após o tx foi o único fator que } \\
\text { afetou a adesão ao acompanhamento (b } \\
\text { = -0,210; } \mathrm{P}<0,05 \text { ). A análise de } \\
\text { regressão linear múltipla mostrou que a } \\
\text { adesão ao acompanhamento, o nível } \\
\text { econômico, a situação profissional, a } \\
\text { fonte de doadores e a doença original } \\
\text { afetaram a QV. }\end{array}$ & $\begin{array}{l}\text { The Quality of Life } \\
\text { of Kidney } \\
\text { Transplantation } \\
\text { Recipients } \\
\text { Questionnaire }\end{array}$ \\
\hline $\begin{array}{l}\text { ScienceDi } \\
\text { rect }\end{array}$ & $\begin{array}{l}\text { Avaliação da } \\
\text { qualidade de } \\
\text { vida e gravidade } \\
\text { da depressão, } \\
\text { ansiedade e } \\
\text { estresse em } \\
\text { pacientes após } \\
\text { transplante renal } \\
\text { (Czyżewski et } \\
\text { al., 2018) }\end{array}$ & 2018 & Polônia & $\begin{array}{l}\text { Avaliar a QV em } \\
\text { receptores de KTx. }\end{array}$ & $\begin{array}{l}\text { A análise de correlação mostrou uma } \\
\text { influência estatisticamente significativa } \\
\text { da idade na saúde geral funcionamento } \\
\text { físico e avaliação da saúde física geral. } \\
\text { Avaliou se o pós-tx em } 1,10 \text { e mais de } \\
10 \text { anos. A gravidade média da } \\
\text { depressão, ansiedade e estresse entre os } \\
\text { indivíduos mudou ao longo do tempo } \\
\text { desde o KTx, mostrando -se altas no } \\
\text { primeiro ano e após os } 10 \text { anos e } \\
\text { reduzida com } 10 \text { anos }\end{array}$ & $\begin{array}{l}\text { Short Form } 36 \\
\text { Health Survey }\end{array}$ \\
\hline BVS & $\begin{array}{l}\text { Validação do } \\
\text { Sistema de } \\
\text { Informação de } \\
\text { Medição de } \\
\text { Resultados } \\
\text { Relatados pelo } \\
\text { Paciente } \\
\text { (PROMIS) -57 e } \\
\text {-29 item formas } \\
\text { abreviadas entre } \\
\text { receptores de } \\
\text { transplante renal. } \\
\text { (Tang et al., } \\
\text { 2019) }\end{array}$ & 2019 & Canadá & $\begin{array}{l}\text { O PROMIS) visa } \\
\text { abordar a falta de } \\
\text { medidas generalizáveis } \\
\text { e universais dos PROM } \\
\text { para avaliar a QVRS. } \\
\text { Não foi validado para } \\
\text { pacientes com DRC. } \\
\text { Nosso objetivo é } \\
\text { validar os questionários } \\
\text { PROMIS-57 e } \\
\text { PROMIS-29 entre } \\
\text { receptores de } \\
\text { transplante renal. }\end{array}$ & $\begin{array}{l}\text { A idade média dos } 177 \text { participantes foi } \\
\text { de } 50,57 \% \text { eram do sexo masculino e } \\
55 \% \text { caucasianos. A consistência interna } \\
\text { de cada domínio foi alta ( } \alpha \text { de } \\
\text { Cronbach }>0,88 \text { ). A análise fatorial } \\
\text { confirmatória mostrou boa validade } \\
\text { estrutural para a maioria dos domínios } \\
\text { (CFI> } 0,95, \text { RMSEA }<0,05) \text {. A } \\
\text { confiabilidade teste-reteste indicou boa } \\
\text { concordância (ICC }>0,6) \text {. }\end{array}$ & $\begin{array}{l}\text { PROMIS-57, } \\
\text { PROMIS-29, } \\
\text { Questionário de } \\
\text { Saúde do Paciente } \\
\text { (PHQ9), } \\
\\
\text { Transtorno de } \\
\text { Ansiedade Geral ( } \\
\text { GAD7), } \\
\text { Escala de Avaliação } \\
\text { de Sintomas de } \\
\text { Edmonton revisada } \\
\text { (ESASr), } \\
\text { Qualidade de Vida } \\
\text { na Doença Renal } \\
\text { (KDQoL-36) }\end{array}$ \\
\hline Pubmed & $\begin{array}{l}\text { Estabelecendo } \\
\text { uma medida } \\
\text { essencial para } \\
\text { participação da } \\
\text { vida. Resultados } \\
\text { padronicados em } \\
\text { Transplante } \\
\text { renal. Relatório } \\
\text { de Consenso de } \\
\text { um Workshop } \\
\text { (Ju et al., 2019) }\end{array}$ & 2019 & Austrália & $\begin{array}{l}\text { Descrever as } \\
\text { perspectivas de } \\
\text { pacientes, cuidadores e } \\
\text { profissionais de saúde } \\
\text { em estabelecer uma } \\
\text { medida para a } \\
\text { participação de vida, a } \\
\text { serem usadas em todos } \\
\text { os ensaios que } \\
\text { envolvem RTX }\end{array}$ & $\begin{array}{l}\text { A participação da vida é um resultado } \\
\text { criticamente importante para os } \\
\text { receptores de transplante renal e } \\
\text { profissionais de saúde. Embora exista } \\
\text { uma ampla gama de atividades, os } \\
\text { pacientes preferiram interpretar isso } \\
\text { com base em seus próprios valores, } \\
\text { objetivos e prioridades. }\end{array}$ & $\begin{array}{l}\text { Short Form } 36 \\
\text { Health Survey; } \\
\text { EuroQol-5D (EQ- } \\
\text { 5D), }\end{array}$ \\
\hline $\begin{array}{l}\text { ScienceDi } \\
\text { rect }\end{array}$ & $\begin{array}{l}\text { Perfis de } \\
\text { aderência em } \\
\text { pacientes com } \\
\text { transplante renal: } \\
\text { causas e } \\
\text { conseqüências } \\
\text { (Villeneuve et } \\
\text { al., 2020) }\end{array}$ & 2020 & França & $\begin{array}{l}\text { Explorar a adesão em } \\
\text { pacientes } \\
\text { transplantados renais } \\
\text { acompanhados por até } \\
\text { três anos após o } \\
\text { transplante }\end{array}$ & $\begin{array}{l}\text { A classe aderente representou } 85 \% \text { e os } \\
\text { não aderentes, menor adesão em um } \\
\text { mês, piorando com o tempo. Boa adesão } \\
\text { foi associada com idade > } 50 \text { anos, } \\
\text { menos episódios de depressão e um } \\
\text { melhor componente de saúde mental da } \\
\text { qualidade de vida. A sobrevivência sem } \\
\text { episódios de rejeição aguda foi maior na } \\
\text { classe aderente }\end{array}$ & $\begin{array}{l}\text { Short Form } 36 \\
\text { Health Survey }\end{array}$ \\
\hline
\end{tabular}


Research, Society and Development, v. 10, n. 13, e337101321548, 2021

(CC BY 4.0) | ISSN 2525-3409 | DOI: http://dx.doi.org/10.33448/rsd-v10i13.21548

\begin{tabular}{|c|c|c|c|c|c|c|}
\hline $\begin{array}{l}\text { ScienceDi } \\
\text { rect }\end{array}$ & $\begin{array}{l}\text { A qualidade de } \\
\text { vida e a } \\
\text { satisfação mental } \\
\text { melhoram } \\
\text { lentamente no } \\
\text { transplante renal } \\
\text { preemptivo em } \\
\text { comparação com } \\
\text { o transplante } \\
\text { renal não } \\
\text { preemptivo. } \\
\text { (Mitsui et al., } \\
\text { 2020) }\end{array}$ & 2020 & Japão & $\begin{array}{l}\text { Examinar as diferenças } \\
\text { na qualidade de vida } \\
\text { entre receptores de } \\
\text { PEKT e não-PEKT. }\end{array}$ & $\begin{array}{l}\text { Oitenta e oito pacientes foram } \\
\text { submetidos a TX renal de doador vivo. } \\
\text { No grupo não-PEKT houve melhora nos } \\
\text { escores dos domínios físico e mental de } \\
3 \text { meses até } 12 \text { pós tx. Já o grupo PEKT, } \\
\text { apenas o escore do dom. físico e mental } \\
\text { melhorou em } 3 \text { meses, e funcionamento } \\
\text { social melhorou em } 12 \text { meses. }\end{array}$ & $\begin{array}{l}\text { Short Form } 36 \\
\text { Health Survey }\end{array}$ \\
\hline Pubmed & $\begin{array}{l}\text { Mudanças na } \\
\text { (QV) e das } \\
\text { (PROM) nos } \\
\text { receptores de tx } \\
\text { renal, de doador } \\
\text { vivo e doador } \\
\text { falecido, e que } \\
\text { aguardam o tx } \\
\text { no programa } \\
\text { Attom do Reino } \\
\text { Unido: uma } \\
\text { coorte } \\
\text { longitudinal } \\
\text { (Gibbons et al., } \\
\text { 2021) }\end{array}$ & 2021 & $\begin{array}{l}\text { Reino } \\
\text { Unido }\end{array}$ & $\begin{array}{l}\text { Examinar a qualidade } \\
\text { de vida }(\mathrm{QV}) \text { e outras } \\
\text { medidas de resultados } \\
\text { relatadas pelo paciente } \\
\text { (PROM) em receptores } \\
\text { de transplante renal e } \\
\text { aqueles que aguardam } \\
\text { transplante. }\end{array}$ & $\begin{array}{l}\text { Em geral, os participantes perceberam } \\
\text { seu transplante como forma de retornar } \\
\text { ao "normal". Ambos os grupos de } \\
\text { transplante relataram melhorias físicas, } \\
\text { redução das restrições dietéticas e } \\
\text { fluidas e melhora do estilo de vida no } \\
\text { pós-transplante. }\end{array}$ & $\begin{array}{l}\text { RDQoL } \\
\text { questionnaire } \\
\text { Well- being } \\
\text { Questionnaire (W- } \\
\text { BQ12), EQ- 5D- 5L }\end{array}$ \\
\hline $\begin{array}{l}\text { ScienceDi } \\
\text { rect }\end{array}$ & $\begin{array}{l}\text { Qualidade de } \\
\text { vida relacionada } \\
\text { à saúde em } \\
\text { receptores de } \\
\text { transplante de } \\
\text { rim idosos: um } \\
\text { estudo de coorte } \\
\text { nacional de } \\
\text { resultados de } \\
\text { curto e longo } \\
\text { prazo (Tsarpali } \\
\text { et al., 2021) }\end{array}$ & 2021 & Noruega & $\begin{array}{l}\text { Avaliar } \\
\text { prospectivamente as } \\
\text { mudanças na QVRS } \\
\text { desde o alistamento até } \\
3 \text { anos após o } \\
\text { transplante e examinar } \\
\text { os preditores pré- } \\
\text { transplante dos } \\
\text { resultados pós- } \\
\text { transplante. }\end{array}$ & $\begin{array}{l}\text { Entre } 289 \text { os pacientes, } 220 \text { (idade } \\
\text { média de } 71,5 \text { anos) sofreram tx e } 136 \\
\text { completaram o acompanhamento de } \\
\text { QVRS de } 3 \text { anos. A QVRS pós-tx, tanto } \\
\text { genérica quanto específica para rins, } \\
\text { melhorou substancialmente e o } \\
\text { benefício persistiu por } 3 \text { anos. Os } \\
\text { pacientes diáliticos por } \geq 1 \text { ano com } \\
\text { pontuação > } 7 \text { no pré-tx } \\
\text { experimentaram uma deterioração física } \\
\text { acentuada e sustentada após o } \\
\text { transplante. }\end{array}$ & KDQOL-SF \\
\hline
\end{tabular}

Fonte: Autores.

Caracterizando a amostra selecionada de 23 estudos, observou-se que, desde o ano de 1996, existem publicações referentes ao tema MRRP, bem como questionários que mensuram a qualidade de vida em transplantados renais. Apesar de mostrar uma constância, verificou-se uma baixa produção científica de estudos dessa natureza, com média de 1,5 publicações por anos, dentro do intervalo da amostra (1996-2021). Confirmou-se também um equilíbrio em relação à distribuição de publicações por ano, no qual apenas o ano de 2006 se destacou com quatro estudos (17,39\%).

Dentre os países que realizaram estudos na temática abordada, destacaram-se os Estados Unidos com cinco estudos (21,7\%). O continente europeu aglutinou 11 estudos (47,8\%), seguido do continente asiático com três (13\%). O Brasil obteve somente um (01) estudo publicado (4,3\%) em 2003, divergindo consideravelmente do volume de publicações internacionais.

Quanto aos tipos de estudo, o desenho observacional obteve a maior frequência com dez publicações (41,6\%), seguido dos estudos de coorte com seis $(25 \%)$ e do transversal com quatro (16,7\%). Considerando ainda, a inexistência de um questionário específico para avaliar a QV em transplantados renais observada na literatura, a proposta de validação e adaptação cultural de questionários foi identificada em apenas dois estudos $(8,4 \%)$. 
No aspecto metodológico, encontraram-se publicações eminentemente qualitativas, que utilizaram a pesquisa exploratório-descritiva. Observou-se que os estudos aplicaram questionários de QV em uma população de pacientes préselecionada, na qual os resultados foram coletados para análise. A descrição das dimensões dos questionários ainda se perfaz pouco explorada, bem como as dimensões físicas e mentais relacionadas à QV, aparecendo em baixa frequência nos resultados dos estudos.

Foram identificados 30 questionários diferentes nos estudos apontados nesta pesquisa. Ressalta-se que houve utilização de mais de um questionário na maior parte dos estudos, demonstrando a ampla diversidade e ao mesmo tempo, a falta de uniformidade na aplicação de instrumentos que avaliam QV em pacientes transplantados renais, em todo o mundo.

$\mathrm{Na}$ amostra desta pesquisa houve o predomínio do questionário Health Survey Short-Form 36 (SF-36), identificado dez vezes (35,7\%). Outros questionários como o Kidney Disease and Quality of Life-Short Form (KDQOL-SF) e Perfil de Impacto da Doença foram identificados duas vezes cada $(7,14 \%)$. O restante dos questionários apareceu apenas uma vez, sendo aglutinados como outros $14(50 \%)$.

As questões mais abordadas nos estudos se concentraram no tempo de acompanhamento no pós-transplante, variando entre menos de um ano, três anos, dez anos e mais de dez anos; a percepção da autoimagem, capacidade funcional, enfrentamento do estresse, depressão e ansiedade, experiência do transplante, situação socioeconômica (SES), qualidade de vida relacionada à saúde (QVRS), uso de imunossupressores e adesão ao tratamento.

\section{Discussão}

Com o avanço das técnicas cirúrgicas, da farmacotécnica de imunossupressão e do manejo do transplante renal, a longevidade de indivíduos pós-transplante aumentou, de acordo com os dados levantados neste estudo (Lim et al., 2017; Muehrer \& Becker, 2005; Sharp, 1995).

Desta forma, a preocupação dos profissionais da saúde que lidam com o transplante agregou um caráter interdisciplinar, pois a sobrevida e a não-rejeição do enxerto, passaram a integrar diversos saberes, entre vários outros parâmetros importantes. Entre tais parâmetros, destacou-se a QV, pois, em sequência à preocupação inicial e expectativa do resultado positivo do transplante, existe a ansiedade do paciente para se reintegrar à sua nova vida, e isto se relaciona de formas diferentes, entre as diferentes pessoas (Muehrer \& Becker, 2005; Sharp, 1995).

A diversidade de características dos pacientes submetidos a um transplante e a maneira como cada pessoa enfrenta a doença, criam múltiplas variáveis que dificultam a mensuração da qualidade de vida de forma individual(Wu et al., 2010). Isso foi constatado nesta pesquisa, dada a variedade de questões e aspectos que foram abordadas nos estudos, por diversos questionários.

Este estudo encontrou artigos com abordagem do MRRP desde 1996. Diante disso, inferiu-se que pesquisas com abordagem de questionários que avaliam a QV, considerando os relatos do paciente coincidiram com a nova concepção de saúde e QV pela Organização Mundial da Saúde (OMS), publicada em 1995. Estas foram entendidas como "um estado de bem-estar físico, mental e social completo e não apenas a ausência de doença ou enfermidade" (The World Health Organization Quality of Life Assessment (WHOQOL), 1995), gerando inquietações a respeito de como mensurá-las.

Sendo assim, as mudanças conceituais compuseram um ganho de maior espaço, na orientação de tratamentos, principalmente na abordagem das doenças crônicas. Aliado a isto, trouxe a percepção de QV, para um novo patamar, colocando o paciente na centralidade do processo (Wu \& Predmore, 2019a). Desta forma, as relações do indivíduo com suas próprias expectativas, padrões e preocupações passaram a ser importantes no contexto da saúde, do bem-estar físico e psicológico (Kamran \& Fife-Schaw, 2012). 
Neste trabalho foi possível observar que algumas pesquisas avaliaram a qualidade de vida com recortes de tempo, considerando a fase pré-procedimento, ainda na fase dialítica, bem como o pós-operatório imediato com períodos de observação que variaram entre seis meses até um ano, e, a qualidade de vida no pós-transplante. O último período englobou intervalos de 1-5 anos, 5-10 anos e mais de dez anos e mostrou-se o mais abordado, pois mostraram melhora na saúde geral dos pacientes com o passar do tempo, e mesmo sintomas como depressão, ansiedade e stress, se alteram com o passar dos anos.

De maneira geral, existe uma clara relação entre o transplante renal e a qualidade de vida, de acordo com a literatura revisada. Esta melhora pode ser percebida nos primeiros seis meses após o transplante. Vale salientar que o pós-operatório imediato e os primeiros meses após a cirurgia, são fases decisivas para determinar o sucesso do transplante(Baker et al., 2017; Graziadei, 2014; See et al., 2018). Tais períodos impõem à pessoa recém-transplantada a adaptação ao novo órgão, ao esquema de imunossupressão, e uma nova rotina de vida (Baker et al., 2017; Flechner, 2016; Lim et al., 2017), sendo de muita importância em seu processo de recuperação.

O pós-transplante tardio visa o alcance da melhoria na qualidade de vida, desde que as condições clínicas, laboratoriais e de imunossupressão, estejam controladas (Adeeb et al., 2021; Alvares et al., 2012; Baker et al., 2017). Pode-se inferir que a qualidade de vida, muitas vezes, é concebida como um parâmetro secundário na maioria das pesquisas nesta área, provavelmente devido à grande preocupação em controlar todas as possíveis causas de insucesso, em um procedimento tão complexo como o transplante renal (Kamran \& Fife-Schaw, 2012; Wasson et al., 1992). Parâmetros de atividade física, que podem se relacionar com a autonomia e condições de mobilidade também são importantes para a percepção de qualidade de vida neste público-alvo.

Essa revisão constatou a utilização de diferentes instrumentos para avaliação da QV em transplantados renais. Alguns instrumentos já possuem validação científica e são utilizados em amplo espectro no contexto internacional, além da existência de questionários desenvolvidos para uso local, considerando as questões culturais e epidemiológicas.

Os questionários divergiram entre si, considerando variáveis como número de itens e dimensões abordadas, as quais transitaram pela falta de consenso, até o momento, acerca da mensuração de qualidade de vida. Entretanto, é válido ressaltar que não ainda não há instrumentos específicos para a avaliação da QV no pós-transplante renal.

O Health Survey Short Form 36 (SF-36) foi identificado como o questionário mais utilizado entre os estudos englobados nesta pesquisa(Monica \& California 90401-3208, [s.d.]). Trata-se de um instrumento de abordagem genérica, composto por 36 itens que avaliam as seguintes dimensões: capacidade funcional, desempenho das atividades diárias, como capacidade de cuidar de si, vestir-se, tomar banho e subir escadas; aspectos físicos (impacto da saúde física no desempenho das atividades diárias e ou profissionais); dor (nível de dor e o impacto no desempenho das atividades diárias e ou profissionais); estado geral de saúde (percepção subjetiva do estado geral de saúde); vitalidade (percepção subjetiva do estado de saúde); aspectos sociais (reflexo da condição de saúde física nas atividades sociais); aspectos emocionais (reflexo das condições emocionais no desempenho das atividades diárias e ou profissionais) e saúde mental (escala de humor e bem-estar).

Um estudo realizado na China (2019) revelou o SF-36 como uma das primeiras abordagem MRRP a ser utilizado em pessoas com Doença Renal Crônica (Kurtin et al., 1992). Desde então, houve um aumento do volume de instrumentos utilizados, sendo que hoje, já são mais de 23 que podem ser aplicados na fase de diálise. Dentre os artigos que integraram esta revisão, o SF-36 foi utilizado na avaliação da qualidade de vida, antes e após o transplante. Entretanto, as dimensões avaliadas sofreram divergências, condicionadas ao interesse do avaliador e de sua pesquisa.

O Kidney Disease and Quality of Life-Short Form (KDQOL-SF) foi um instrumento encontrado em proporção bastante inferior, comparado ao SF-36. Assim, ainda merece destaque por se tratar de instrumento autoaplicável em indivíduos com doença renal e em diálise(Yapa et al., 2020). 
Neste contexto é válido destacar o uso da MRRP, pois tal mensuração se configura como mais uma ferramenta importante na coleta de percepções diretamente dos indivíduos transplantados e, como isto tem impactado suas vidas (Wu \& Predmore, 2019b). A então ferramenta confere uma maior identidade ao transplantado, durante seu acompanhamento nesta fase, pois os dados são coletados diretamente deste, no que concerne aos aspectos físicos, psicológicos e sociais, e como isto se relaciona com as suas atividades diárias.

A análise desta revisão (Quadro 1), permitiu verificar um amplo espectro, que circunda o tema da QV e do transplante renal. A falta de instrumentos mais específicos, centraliza nos pesquisadores os aspectos prioritários em relação à QV em transplantados renais, fato que reduz as possibilidades de uma avaliação centrada no paciente (Flythe et al., 2015)

O uso da MRRP caminha adquirindo maior importância, pois permite uma abordagem compartilhada entre os profissionais da saúde e os pacientes, visando o progresso no tratamento e com isso provocar mudanças reais de comportamento e satisfação. Entende-se que para o transplante, a adesão é um dos pontos mais importantes para o sucesso do tratamento, mas deve-se considerar que os tratamentos da atualidade, não priorizam apenas o tempo. Os avanços na área da saúde mostram de forma mais incisiva, a importância de "como" se vive este tempo a mais.

\section{Conclusão}

Esta pesquisa não reconheceu, até o momento, instrumentos que possam ser aplicados para avaliar a QV em pacientes transplantados renais, de uma forma específica. Apesar do SF-36 ter sido apontado como o questionário mais utilizado na literatura internacional, ele ainda possui uma abordagem genérica, o que estabelece uma baixa sensibilidade da percepção da qualidade de vida pelo paciente.

Foi possível identificar que as dimensões, domínios e itens dispostos no questionário SF-36 deixaram de capturar nuances específicas do paciente transplantado renal. É válido ressaltar que os demais questionários encontrados nesse estudo foram oriundos do SF-36, o que denota generalização do objeto a ser avaliado. Nessa direção inferiu-se que a utilização de questionários genéricos não assegura uma análise mais relevante da qualidade de vida, sendo pertinente abordar a QV do ponto de vista do paciente que recebeu um transplante renal.

Por fim, este estudo apontou para a necessidade de novas pesquisas que avaliem a aplicação de instrumentos mais específicos para esta população, com vistas a captar as reais necessidades de um transplantado renal, contribuindo para uma abordagem holística e equânime desses pacientes.

\section{Referências}

Adeeb, H., Ullah, I., Zeb, M., \& Haq, M. (2021). Clinical factors and comorbidities affecting health-related quality of life in postrenal transplant patients. Porto Biomedical Journal, 6(3), e131. https://doi.org/10.1097/j.pbj.0000000000000131

Agochukwu, N. Q., Wittmann, D., Boileau, N. R., Dunn, R. L., Montie, J., Kim, T., Miller, D. C., Peabody, J., \& Carlozzi, N. E. (2020). Reply to K.P. Weinfurt et al. Journal of Clinical Oncology, 38(6), 654-655. https://doi.org/10.1200/JCO.19.02642

Alvares, J., Cesar, C. C., Acurcio, F. de A., Andrade, E. I. G., \& Cherchiglia, M. L. (2012). Quality of life of patients in renal replacement therapy in Brazil: Comparison of treatment modalities. Quality of Life Research: An International Journal of Quality of Life Aspects of Treatment, Care and Rehabilitation, 21(6), 983-991. https://doi.org/10.1007/s11136-011-0013-6

Baker, R. J., Mark, P. B., Patel, R. K., Stevens, K. K., \& Palmer, N. (2017). Renal association clinical practice guideline in post-operative care in the kidney transplant recipient. BMC Nephrology, 18(1), 174. https://doi.org/10.1186/s12882-017-0553-2

Barotfi, S., Molnar, M. Zs., Almasi, C., Kovacs, A. Zs., Remport, A., Szeifert, L., Szentkiralyi, A., Vamos, E., Zoller, R., Eremenco, S., Novak, M., \& Mucsi, I. (2006). Validation of the Kidney Disease Quality of Life-Short Form questionnaire in kidney transplant patients. Journal of Psychosomatic Research, 60(5), 495-504. https://doi.org/10.1016/j.jpsychores.2005.09.009

Cetingok, M., Winsett, R. P., \& Hathaway, D. K. (2004). A comparative study of quality of life among the age groups of kidney transplant recipients. Progress in Transplantation (Aliso Viejo, Calif.), 14(1), 33-38. https://doi.org/10.7182/prtr.14.1.g5612w70x8331k61

Czyżewski, Ł., Frelik, P., Wyzgał, J., \& Szarpak, Ł. (2018). Evaluation of Quality of Life and Severity of Depression, Anxiety, and Stress in Patients After Kidney Transplantation. Transplantation Proceedings, 50(6), 1733-1737. https://doi.org/10.1016/j.transproceed.2018.04.026 
Dobbels, F., Wong, S., Min, Y., Sam, J., \& Kalsekar, A. (2014). Beneficial effect of belatacept on health-related quality of life and perceived side effects: Results from the BENEFIT and BENEFIT-EXT trials. Transplantation, 98(9), 960-968. https://doi.org/10.1097/TP.0000000000000159

Flechner, S. M. (2016). Optimizing immunosuppression: Who can do more with less? Transplant International: Official Journal of the European Society for Organ Transplantation, 29(1), 20-22. https://doi.org/10.1111/tri.12676

Flythe, J. E., Powell, J. D., Poulton, C. J., Westreich, K. D., Handler, L., Reeve, B. B., \& Carey, T. S. (2015). Patient-Reported Outcome Instruments for Physical Symptoms Among Patients Receiving Maintenance Dialysis: A Systematic Review. American Journal of Kidney Diseases, 66(6), 1033-1046. https://doi.org/10.1053/j.ajkd.2015.05.020

Franke, G. H., Yücetin, L., Yaman, H., Reimer, J., \& Demirbas, A. (2006). Disease-Specific Quality of Life in Turkish Patients After Successful Kidney Transplantation. Transplantation Proceedings, 38(2), 457-459. https://doi.org/10.1016/j.transproceed.2005.12.110

Gibbons, A., Bayfield, J., Cinnirella, M., Draper, H., Johnson, R. J., Oniscu, G. C., Ravanan, R., Tomson, C., Roderick, P., Metcalfe, W., Forsythe, J. L. R., Dudley, C., Watson, C. J. E., Bradley, J. A., \& Bradley, C. (2021). Changes in quality of life (QoL) and other patient-reported outcome measures (PROMs) in living-donor and deceased-donor kidney transplant recipients and those awaiting transplantation in the UK ATTOM programme: A longitudinal cohort questionnaire survey with additional qualitative interviews. BMJ Open, 11(4), e047263. https://doi.org/10.1136/bmjopen-2020-047263

Graziadei, I. (2014). [Intensive care treatment before and after liver transplantation]. Medizinische Klinik, Intensivmedizin Und Notfallmedizin, 109(6), 411417. https://doi.org/10.1007/s00063-014-0364-1

Johnson, C. D., Wicks, M. N., Milstead, J., Hartwig, M., \& Hathaway, D. K. (1998). Racial and gender differences in quality of life following kidney transplantation. Image--the Journal of Nursing Scholarship, 30(2), 125-130. https://doi.org/10.1111/j.1547-5069.1998.tb01266.x

Ju, A., Josephson, M. A., Butt, Z., Jowsey-Gregoire, S., Tan, J., Taylor, Q., Fowler, K., Dobbels, F., Caskey, F., Jha, V., Locke, J., Knoll, G., Ahn, C., Hanson, C. S., Sautenet, B., Manera, K., Craig, J. C., Howell, M., Rutherford, C., ... Patients and family members. (2019). Establishing a Core Outcome Measure for Life Participation: A Standardized Outcomes in Nephrology-kidney Transplantation Consensus Workshop Report. Transplantation, 103(6), 1199-1205. https://doi.org/10.1097/TP.0000000000002476

Kamran, F., \& Fife-Schaw, C. (2012). Psychosocial versus clinical factors influencing QoL among renal transplant recipients. International Journal of Research Studies in Psychology, 2. https://doi.org/10.5861/ijrsp.2012.159

Kizilisik, A. T., Feurer, I. D., VanBuren, D. H., Wise, P., Van Buren, D., Hopkins, J., Ray, J., Nylander, W., Shaffer, D., Helderman, J. H., Langone, A. J., Speroff, T., \& Pinson, C. W. (2003). Effects of diabetes and cadaveric organs on functional performance and health-related quality of life after kidney transplantation. The American Journal of Surgery, 186(5), 535-539. https://doi.org/10.1016/j.amjsurg.2003.07.013

Kong, I. L. L., \& Molassiotis, A. (1999). Quality of life, coping and concerns in Chinese patients after renal transplantation. International Journal of Nursing Studies, 36(4), 313-322. https://doi.org/10.1016/S0020-7489(99)00025-5

Kurtin, P. S., Davies, A. R., Meyer, K. B., DeGiacomo, J. M., \& Kantz, M. E. (1992). Patient-Based Health Status Measures in Outpatient Dialysis: Early Experiences in Developing an Outcomes Assessment Program. Medical Care, 30(5), MS136-MS149.

Laupacis, A., Keown, P., Pus, N., Krueger, H., Ferguson, B., Wong, C., \& Muirhead, N. (1996). A study of the quality of life and cost-utility of renal transplantation. Kidney International, 50(1), 235-242. https://doi.org/10.1038/ki.1996.307

Liberati, A., Altman, D. G., Tetzlaff, J., Mulrow, C., Gøtzsche, P. C., Ioannidis, J. P. A., Clarke, M., Devereaux, P. J., Kleijnen, J., \& Moher, D. (2009). The PRISMA statement for reporting systematic reviews and meta-analyses of studies that evaluate healthcare interventions: Explanation and elaboration. BMJ (Clinical Research Ed.), 339, b2700. https://doi.org/10.1136/bmj.b2700

Lim, M. A., Kohli, J., \& Bloom, R. D. (2017). Immunosuppression for kidney transplantation: Where are we now and where are we going? Transplantation Reviews (Orlando, Fla.), 31(1), 10-17. https://doi.org/10.1016/j.trre.2016.10.006

Mitsui, Y., Araki, M., Maruyama, Y., Yoshinaga, K., Sadahira, T., Wada, K., Tanabe, K., Kitagawa, M., Kobayashi, Y., Watanabe, M., Watanabe, T., \& Nasu, Y. (2020). Quality of Life and Mental Satisfaction Improve Slowly in Preemptive Kidney Transplantation Compared With Nonpreemptive Kidney Transplantation. Transplantation Proceedings, 52(3), 740-747. https://doi.org/10.1016/j.transproceed.2020.01.042

Monica, 1776 Main Street Santa, \& California 90401-3208. ([s.d.]). 36-Item Short Form Survey Instrument (SF-36). Recuperado 2 de setembro de 2021, de https://www.rand.org/health-care/surveys_tools/mos/36-item-short-form/survey-instrument.html

Muehrer, R. J., \& Becker, B. N. (2005). Life after transplantation: New transitions in quality of life and psychological distress. Seminars in Dialysis, 18(2), 124-131. https://doi.org/10.1111/j.1525-139X.2005.18214.x

Ponton, P., Rupolo, G. P., Marchini, F., Feltrin, A., Perin, N., Mazzoldi, M. A., Giacon, B., Baldan, N., \& Rigotti, P. (2001). Quality-of-life change after kidney transplantation. Transplantation Proceedings, 33(1), 1887-1889. https://doi.org/10.1016/S0041-1345(00)02737-8

See, S. B., Aubert, O., Loupy, A., Veras, Y., Lebreton, X., Gao, B., Legendre, C., Anglicheau, D., \& Zorn, E. (2018). Post-Transplant Natural Antibodies Associate with Kidney Allograft Injury and Reduced Long-Term Survival. Journal of the American Society of Nephrology: JASN, 29(6), 1761-1770. https://doi.org/10.1681/ASN.2017111157

Sesso, R., Rodrigues-Neto, J. F., \& Ferraz, M. B. (2003). Impact of socioeconomic status on the quality of life of ESRD patients. American Journal of Kidney Diseases, 41(1), 186-195. https://doi.org/10.1053/ajkd.2003.50003

Shah, V. S., Ananth, A., Sohal, G. K., Bertges-Yost, W., Eshelman, A., Parasuraman, R. K., \& Venkat, K. K. (2006). Quality of Life and Psychosocial Factors in Renal Transplant Recipients. Transplantation Proceedings, 38(5), 1283-1285. https://doi.org/10.1016/j.transproceed.2006.03.027 
Sharp, L. A. (1995). Organ transplantation as a transformative experience: Anthropological insights into the restructuring of the self. Medical Anthropology Quarterly, 9(3), 357-389. https://doi.org/10.1525/maq.1995.9.3.02a00050

Soares, C. B., Hoga, L. A. K., Peduzzi, M., Sangaleti, C., Yonekura, T., \& Silva, D. R. A. D. (2014). Integrative Review: Concepts and Methods Used In Nursing. Revista Da Escola de Enfermagem Da USP, 48(2), 335-345. https://doi.org/10.1590/S0080-6234201400002000020

Tang, E., Ekundayo, O., Peipert, J. D., Edwards, N., Bansal, A., Richardson, C., Bartlett, S. J., Howell, D., Li, M., Cella, D., Novak, M., \& Mucsi, I. (2019). Validation of the Patient-Reported Outcomes Measurement Information System (PROMIS)-57 and -29 item short forms among kidney transplant recipients. Quality of Life Research: An International Journal of Quality of Life Aspects of Treatment, Care and Rehabilitation, $28(3)$, 815-827. https://doi.org/10.1007/s11136-018-2058-2

The World Health Organization Quality of Life assessment (WHOQOL): Position paper from the World Health Organization. (1995). Social Science \& Medicine (1982), 41(10), 1403-1409. https://doi.org/10.1016/0277-9536(95)00112-k

Tsarpali, V., Midtvedt, K., Lønning, K., Bernklev, T., Lippe, N. von der, Reisæter, A. V., Brunborg, C., \& Heldal, K. (2021). Health-Related Quality of Life in Older Kidney Transplant Recipients: A National Cohort Study of Short- and Longer-Term Outcomes. Kidney Medicine. https://doi.org/10.1016/j.xkme.2021.05.007

Urstad, K. H., Øyen, O., Andersen, M. H., Moum, T., \& Wahl, A. K. (2012). The effect of an educational intervention for renal recipients: A randomized controlled trial. Clinical Transplantation, 26(3), E246-253. https://doi.org/10.1111/j.1399-0012.2012.01666.x

Verberne, W. R., Das-Gupta, Z., Allegretti, A. S., Bart, H. A. J., van Biesen, W., García-García, G., Gibbons, E., Parra, E., Hemmelder, M. H., Jager, K. J., Ketteler, M., Roberts, C., Al Rohani, M., Salt, M. J., Stopper, A., Terkivatan, T., Tuttle, K. R., Yang, C.-W., Wheeler, D. C., \& Bos, W. J. W. (2019). Development of an International Standard Set of Value-Based Outcome Measures for Patients With Chronic Kidney Disease: A Report of the International Consortium for Health Outcomes Measurement (ICHOM) CKD Working Group. American Journal of Kidney Diseases, 73(3), 372-384. https://doi.org/10.1053/j.ajkd.2018.10.007

Villeneuve, C., Rousseau, A., Rerolle, J.-P., Couzi, L., Kamar, N., Essig, M., Etienne, I., Westeel, P.-F., Büchler, M., Esposito, L., Thierry, A., Marquet, P., \& Monchaud, C. (2020). Adherence profiles in kidney transplant patients: Causes and consequences. Patient Education and Counseling, 103(1), 189-198. https://doi.org/10.1016/j.pec.2019.08.002

Von der Lippe, N., Waldum, B., Brekke, F. B., Amro, A. A. G., Reisæter, A. V., \& Os, I. (2014). From dialysis to transplantation: A 5-year longitudinal study on self-reported quality of life. BMC Nephrology, 15, 191. https://doi.org/10.1186/1471-2369-15-191

Ware, J. E., Richardson, M. M., Meyer, K. B., \& Gandek, B. (2019). Improving CKD-Specific Patient-Reported Measures of Health-Related Quality of Life. Journal of the American Society of Nephrology: JASN, 30(4), 664-677. https://doi.org/10.1681/ASN.2018080814

Wasson, J., Keller, A., Rubenstein, L., Hays, R., Nelson, E., Johnson, D., \& Project, T. D. P. C. C. (1992). Benefits and Obstacles of Health Status Assessment in Ambulatory Settings: The Clinician's Point of View. Medical Care, 30(5), MS42-MS49.

Wolfe, R. A., Ashby, V. B., Milford, E. L., Ojo, A. O., Ettenger, R. E., Agodoa, L. Y., Held, P. J., \& Port, F. K. (1999). Comparison of mortality in all patients on dialysis, patients on dialysis awaiting transplantation, and recipients of a first cadaveric transplant. The New England Journal of Medicine, 341(23), 17251730. https://doi.org/10.1056/NEJM199912023412303

Wu, A. W., \& Predmore, Z. S. (2019a). Patient-Reported Outcomes: Toward Better Measurement of Patient-Centered Care in CKD. Journal of the American Society of Nephrology, 30(4), 523-525. https://doi.org/10.1681/ASN.2019020169

Wu, A. W., \& Predmore, Z. S. (2019b). Patient-Reported Outcomes: Toward Better Measurement of Patient-Centered Care in CKD. Journal of the American Society of Nephrology, 30(4), 523-525. https://doi.org/10.1681/ASN.2019020169

Wu, A. W., Snyder, C., Clancy, C. M., \& Steinwachs, D. M. (2010). Adding The Patient Perspective To Comparative Effectiveness Research. Health Affairs, 29(10), 1863-1871. https://doi.org/10.1377/hlthaff.2010.0660

XXVI No 4-Anual. ([s.d.]). ABTO. Recuperado 2 de setembro de 2021, de https://site.abto.org.br/publicacao/xxvi-no-4-anual/

Yapa, H. E., Purtell, L., Chambers, S., \& Bonner, A. (2020). The Relationship Between Chronic Kidney Disease, Symptoms and Health-Related Quality of Life: A Systematic Review. Journal of Renal Care, 46(2), 74-84. https://doi.org/10.1111/jorc.12303

Yildirim, A. (2006). The Importance of Patient Satisfaction and Health-Related Quality of Life After Renal Transplantation. Transplantation Proceedings, 38(9), 2831-2834. https://doi.org/10.1016/j.transproceed.2006.08.162

Zaydfudim, V., Feurer, I. D., Moore, D. R., Moore, D. E., Pinson, C. W., \& Shaffer, D. (2010). Pre-transplant Overweight and Obesity Do Not Affect Physical Quality of Life after Kidney Transplantation. Journal of the American College of Surgeons, 210 (3), 336-344. https://doi.org/10.1016/j.jamcollsurg.2009.11.009

Zhao, L., Yan, J., Yang, G.-L., \& Liu, Y. (2017). A Study on Adherence to Follow-up, Quality of Life, and Associated Factors Among Renal Transplant Recipients in China. Transplantation Proceedings, 49(6), 1285-1290. https://doi.org/10.1016/j.transproceed.2017.03.086 